\title{
Spécialisation des conditions de Manin pour les variétés fibrées au-dessus de l'espace projectif
}

\author{
David Harari
}

\begin{abstract}
Let $X$ be a smooth and projective variety such that $X$ is fibred over the projective space. We give sufficient conditions ensuring that the fibres contain adelic points satisfying Manin-like conditions.
\end{abstract}

\section{RÉSUMÉ}

Soit $X$ une variété (projective et lisse) sur un corps de nombres $k$, fibrée au-dessus de l'espace projectif. On donne des conditions suffisantes pour que les fibres contiennent des points adéliques satisfaisant des conditions de type Manin.

\section{Introduction}

\subsection{Présentation du problème}

Soit $X$ une variété (projective et lisse) sur un corps de nombres $k$, fibrée au-dessus de l'espace projectif $\mathbf{P}_{k}^{n}$. Dans de précédents articles [Har94, Har97], on s'est intéressé à la question suivante : si l'obstruction de Manin au principe de Hasse (respectivement à l'approximation faible) est la seule pour les fibres, est-ce encore le cas pour $X$ ? La réponse est positive sous certaines hypothèses géométriques (voir [Har97, théorème 3.2.1]). L'une des difficultés (l'autre problème majeur étant de contrôler le groupe de Brauer des fibres à partir du groupe de Brauer générique, voir [Har97, théorème 2.3.1]) pour établir ce type d'énoncés est la suivante : soit $B$ un sous-groupe du groupe de Brauer $\operatorname{Br} X_{\eta}$ de la fibre générique; partant d'un point adélique $\left(P_{v}\right)$ de $X$ qui est orthogonal à $B \cap \operatorname{Br} X$ (pour l'accouplement de Brauer-Manin), comment trouver une fibre rationnelle $X_{\theta}$ et un point adélique $\left(P_{v}^{\prime}\right)$ de $X_{\theta}$ qui est orthogonal à la spécialisation $B_{\theta} \subset \operatorname{Br} X_{\theta}$ de $B$ ? Ceci n'est pas évident, notamment parce que $B$ et $B_{\theta}$ peuvent être bien plus gros que $B \cap \operatorname{Br} X$.

Dans les travaux précédents sur ce sujet, on se limitait au cas où $B$ était $\operatorname{Br} X_{\eta}$ tout entier. Mais il arrive fréquemment que la question de l'existence d'un point rationnel dans une fibre $X_{\theta}$ soit liée à une petite partie du groupe de Brauer : typiquement si $X_{\theta}$ est elle-même un fibré en courbes de genre 1, plusieurs travaux antérieurs semblent indiquer que c'est le groupe de Brauer 'vertical' qui joue un rôle particulier. C'est notamment le cas dans le travail fondateur de Colliot-Thélène, Skorobogatov et Swinnerton-Dyer [CSS98], et également dans les articles de Bender et SwinnertonDyer [BS01] et Colliot-Thélène [Col01]. Certains de ces résultats ont été reformulés et complétés par Wittenberg [Wit07, chapitres 1 et 2]. Notons qu'il serait du reste très malaisé dans ce type de situations de travailler avec le groupe de Brauer tout entier parce que $\operatorname{Br} X_{\eta}$ est loin d'être fini modulo les constantes, ce qui fait que les méthodes de [Har94] et [Har97] ne s'appliquent pas telles quelles.

Received 8 February 2006, accepted in final form 24 October 2006.

2000 Mathematics Subject Classification 11G35, $14 \mathrm{G} 25$.

Keywords: Brauer group, Hilbert's irreducibility theorem.

This journal is (C) Foundation Compositio Mathematica 2007. 


\section{HARARI}

Il était donc important d'établir un énoncé général garantissant, avec les notations ci-dessus, l'existence de $\left(P_{v}^{\prime}\right)$ (orthogonal à $\left.B_{\theta}\right)$ à partir de celle de $\left(P_{v}\right)$ (orthogonal à $B \cap \operatorname{Br} X$ ) pour n'importe quel sous-groupe $B$, fini modulo $\operatorname{Br}\left(k\left(\mathbf{P}_{k}^{n}\right)\right)$, de $\operatorname{Br} X_{\eta}$. C'est le but de cet article; son résultat principal (théorème 1 ci-dessous) est un ingrédient crucial dans la preuve par Wittenberg d'un théorème [Wit07, théorème 3.4] garantissant sous certaines conditions l'existence de points adéliques dans 'beaucoup de fibres' pour les familles de variétés au-dessus de $\mathbf{P}_{k}^{n}$, théorème qu'il a ensuite utilisé pour étudier les intersections de deux quadriques dans $\mathbf{P}_{k}^{4}$ et $\mathbf{P}_{k}^{5}$ (voir [Wit07, chapitre 3] et [Wit06]). Il a notamment obtenu le résultat frappant suivant : moyennant l'hypothèse de Schinzel (il s'agit d'une généralisation conjecturale du théorème de la progression arithmétique de Dirichlet, voir par exemple [CSS98]) et la finitude du groupe de Tate-Shafarevitch des courbes elliptiques, toute intersection lisse de deux quadriques dans $\mathbf{P}_{k}^{5}$ satisfait le principe de Hasse. Il paraît raisonnable d'espérer que le théorème 1 trouvera des applications à d'autres classes de variétés.

\subsection{Notations et rappels}

Si $k$ est un corps de nombres, on note $\Omega_{k}$ l'ensemble de ses places. Pour tout complété $k_{v}$ de $k$, on dispose de l'invariant local $j_{v}: \operatorname{Br} k_{v} \rightarrow \mathbf{Q} / \mathbf{Z}$. Si $v$ est non archimédienne, on note $k(v)$ le corps résiduel de $k_{v}$. Pour tout ensemble fini $S$ de places de $k$, on désigne par $\mathcal{O}_{k, S}$ l'anneau des $S$-entiers, c'est-à-dire l'anneau des éléments $x$ de $k$ qui vérifient $v(x) \geqslant 0$ pour toute place $v$ qui n'est ni archimédienne ni dans $S$. On fixe une clôture algébrique $\bar{k}$ de $k$. Pour tout $k$-groupe algébrique commutatif $G$ et tout $k$-schéma $X$, on note $H^{i}(X, G)$ (pour $i \geqslant 0$ ) les groupes de cohomologie étale associés; en particulier les $H^{i}(k, G)=H^{i}(\mathrm{Gal}(\bar{k} / k), G(\bar{k}))$ sont les groupes de cohomologie galoisienne usuels. Si $X$ est un schéma régulier intègre de corps des fonctions $K$ et $m$ un point de codimension 1 de $X$ de corps résiduel parfait $\kappa$, on dispose d'une flèche résidu $\partial_{m}: \operatorname{Br} K \rightarrow H^{1}(\kappa, \mathbf{Q} / \mathbf{Z})$ (voir [Gro68, $\S \S 6$ et 7$]$ ).

Si $X$ est une $k$-variété (i.e. un $k$-schéma séparé de type fini) propre, on désigne par $X\left(\mathbf{A}_{k}\right)=$ $\prod_{v \in \Omega_{k}} X\left(k_{v}\right)$ l'ensemble de ses points adéliques. Pour tout sous-groupe $B$ du groupe de Brauer cohomologique $\operatorname{Br} X=H^{2}\left(X, \mathbf{G}_{m}\right)$ (respectivement de $\operatorname{Br} X / \operatorname{Br} k$ ), on note $X\left(\mathbf{A}_{k}\right)^{B}$ l'ensemble des points adéliques $\left(P_{v}\right)$ de $X$ qui sont orthogonaux à $B$ pour l'accouplement de Brauer-Manin, i.e. qui satisfont $\sum_{v \in \Omega_{k}} j_{v}\left(\alpha\left(P_{v}\right)\right)=0$ pour tout $\alpha \in B$ (la somme ne dépend que de la classe de $\alpha$ modulo $\mathrm{Br} k$; cf [Sko01, $\S 2.5 .2$ ], pour plus de détails). Les lois de réciprocité du corps de classes global impliquent que $X\left(\mathbf{A}_{k}\right)^{\operatorname{Br} X}$ contient l'adhérence dans $X\left(\mathbf{A}_{k}\right)$ de l'ensemble $X(k)$ des points rationnels de $X$, pour la topologie produit des topologies $v$-adiques. On dit qu'une classe de variétés vérifie le principe de Hasse (respectivement l'approximation faible) si pour toute variété $X$ dans cette classe, la condition $X\left(\mathbf{A}_{k}\right) \neq \emptyset$ implique $X(k) \neq \emptyset$ (respectivement $X(k)$ est dense dans $X\left(\mathbf{A}_{k}\right)$ pour la topologie produit des topologies $v$-adiques). Ainsi si $X\left(\mathbf{A}_{k}\right)^{\operatorname{Br} X}=\emptyset$ (respectivement $X\left(\mathbf{A}_{k}\right)^{\operatorname{Br} X} \neq$ $X\left(\mathbf{A}_{k}\right)$ ), on a une obstruction, dite obstruction de Manin, au principe de Hasse (respectivement à l'approximation faible).

Soit $p: X \rightarrow Y$ un morphisme entre deux $k$-variétés intègres et lisses dont on note $X_{\eta}$ la fibre générique. Soit $K$ le corps des fonctions de $Y$. Alors pour tout sous-groupe fini $C$ de $\operatorname{Br} X_{\eta} / \operatorname{Br} K$, il existe un ouvert de Zariski non vide $U$ de $Y$ tel que pour tout point fermé $m$ de $U$, on ait un homomorphisme de spécialisation (cf. [Har94, §3.3]) :

$$
C \rightarrow \operatorname{Br} X_{m} / \operatorname{Br}(k(m))
$$

où $X_{m}$ est la fibre de $p$ en $m$ et $k(m)$ le corps résiduel de $m$. Ici on a noté abusivement $\operatorname{Br} K$, $\operatorname{Br}(k(m))$ les images respectives de $\operatorname{Br} K, \operatorname{Br}(k(m))$ dans $\operatorname{Br} X_{\eta}, \operatorname{Br} X_{m}$.

Une $k$-variété est dite scindée si elle contient un ouvert non vide qui est géométriquement intègre sur $k$ (on peut alors demander que cet ouvert soit lisse). Soit $V$ une variété intègre sur un corps de nombres $k$; un sous-ensemble $H$ de $V(k)$ est dit hilbertien élémentaire s'il existe un ouvert de 


\section{SpÉCiAlisation DES CONDitions DE MANin}

Zariski non vide $U$ de $V$ et un revêtement étale $\rho: U^{\prime} \rightarrow U$ avec $U^{\prime}$ intègre tel que $H$ soit l'ensemble des $k$-points de $U$ en lesquels la fibre de $\rho$ est connexe (i.e. intègre puisqu'elle est lisse); un sousensemble de $V(k)$ est dit hilbertien s'il est intersection finie d'ensembles hilbertiens élémentaires. Nous utiliserons les propriétés suivantes, qui découlent immédiatement de la définition :

(i) si $H_{1}$ et $H_{2}$ sont hilbertiens, alors $H_{1} \cap H_{2}$ est hilbertien ;

(ii) l'ensemble des points rationnels d'un ouvert de Zariski non vide de $V$ est hilbertien ;

(iii) la projection d'un sous-ensemble hilbertien de $\mathbf{A}_{k}^{r} \times \mathbf{A}_{k}^{s}$ sur $\mathbf{A}_{k}^{r}$ est un sous-ensemble hilbertien de $\mathbf{A}_{k}^{r}$.

Nous aurons aussi besoin du lemme suivant.

Lemme 1. Soit $V$ une $k$-variété intègre équipée d'un $k$-morphisme dominant $f$ vers $\mathbf{P}_{k}^{r}$. Soient $H$ un sous-ensemble hilbertien de $V$ et $m$ un point rationnel de $\mathbf{P}_{k}^{r}$ en lequel la fibre $V_{m}$ de $f$ est intègre. Alors, si $H \cap V_{m}(k)$ est non vide, c'est un sous-ensemble hilbertien de $V_{m}(k)$.

Démonstration. Il suffit d'établir l'énoncé quand $H$ est un ensemble hilbertien élémentaire. Par définition, il existe alors un ouvert non vide lisse $W$ de $V$ et un revêtement étale intègre $\rho: W^{\prime} \rightarrow W$ tel que $H$ soit l'ensemble des points de $W(k)$ en lesquels la fibre de $\rho$ est intègre. Soient $W_{m}:=$ $W \cap V_{m}$ et $\rho_{m}: W_{m}^{\prime} \rightarrow W_{m}$ la restriction de $\rho$ à $W_{m}$. On a déjà $W_{m} \neq \emptyset$ car $V_{m}(k) \cap H \subset W_{m}(k)$. D'autre part, au moins l'une des fibres du revêtement $\rho_{m}$ est intègre vu que $W_{m}(k) \cap H=V_{m}(k) \cap H$ est non vide. De ce fait $W_{m}^{\prime}$ est intègre (il est lisse et connexe). Comme $V_{m}(k) \cap H$ est précisément l'ensemble des points rationnels de $W_{m}$ en lesquels $\rho_{m}$ est intègre, c'est par définition un sousensemble hilbertien de $V_{m}(k)$.

\section{3 Énoncé du théorème}

Le résultat principal de cet article est le théorème suivant.

ThÉorème 1. Soient $k$ un corps de nombres et $X$ une $k$-variété projective, lisse et géométriquement intègre. On suppose $X$ munie d'un morphisme $p: X \rightarrow \mathbf{P}_{k}^{n}$ projectif et surjectif dont la fibre générique $X_{\eta}$ est géométriquement intègre sur le corps des fonctions $K$ de $\mathbf{P}_{k}^{n}$. On fait l'hypothèse qu'il existe un fermé $F$ de codimension 2 de $\mathbf{P}_{k}^{n}$ en dehors duquel les fibres de $p$ sont scindées. Soit $B$ un sous-groupe de $\operatorname{Br} X_{\eta}$ contenant $\operatorname{Br} K$, et tel que $B / \operatorname{Br} K$ soit fini, on définit $B^{\prime}:=B \cap \operatorname{Br} X$. Alors si $X\left(\mathbf{A}_{k}\right)^{B^{\prime}} \neq \emptyset$, il existe pour tout sous-ensemble hilbertien $H$ de $\mathbf{P}_{k}^{n}(k)$ un point rationnel $\theta \in H$ tel que

$$
X_{\theta}\left(\mathbf{A}_{k}\right)^{B_{\theta}} \neq \emptyset
$$

où $X_{\theta}$ est la fibre de $p$ en $\theta$ et $B_{\theta} \subset \operatorname{Br} X_{\theta} / \operatorname{Br} k$ est la spécialisation de $B / \operatorname{Br} K$ en $\theta$.

De plus si $\left(Q_{v}\right) \in X\left(\mathbf{A}_{k}\right)^{B^{\prime}}$ et $S_{1}$ est un ensemble fini de places de $k$, alors on peut choisir $\theta$ tel que $X_{\theta}\left(\mathbf{A}_{k}\right)^{B_{\theta}}$ contienne des points $\left(P_{v}\right)$ avec $P_{v}$ arbitrairement proche de $Q_{v}$ pour $v \in S_{1}$.

Remarques. Nous faisons les remarques suivantes.

1. On note abusivement encore $\operatorname{Br} K, \operatorname{Br} k$ les images respectives de $\operatorname{Br} K, \operatorname{Br} k$ dans $\operatorname{Br} X_{\eta}, \operatorname{Br} X_{\theta}$. Si $k(X)$ désigne le corps des fonctions de $X$, on a $\operatorname{Br} X \subset \operatorname{Br} X_{\eta} \subset \operatorname{Br}(k(X))$.

2. Le groupe $B / \operatorname{Br} K$ étant fini, il existe un ouvert de Zariski non vide $U_{0}$ de $\mathbf{P}_{k}^{n}$ tel que pour tout $\theta \in U_{0}$, l'homomorphisme de spécialisation $B / \operatorname{Br} K \rightarrow \operatorname{Br} X_{\theta} / \operatorname{Br} k$ soit bien défini. En particulier on sous-entend dans l'énoncé ci-dessus que $\theta$ appartient à $U_{0}(k) \cap H$ (qui est encore un sous-ensemble hilbertien de $\left.\mathbf{P}_{k}^{n}(k)\right)$; alors $X_{\theta}\left(\mathbf{A}_{k}\right)^{B_{\theta}}$ a bien un sens même si $B_{\theta}$ n'est défini que modulo $\mathrm{Br} k$.

3. Il est important de noter que l'on est souvent amené à appliquer une méthode de fibration (pour établir le principe de Hasse ou l'approximation faible) à des variétés $X$ dont le groupe 


\section{HARARI}

de Brauer est trivial, mais qui sont fibrées en variétés à groupe de Brauer non trivial. Cela signifie qu'on aura alors $B_{\theta}$ plus gros que $B \cap \operatorname{Br} X$. En général, les fibres ne satisfont alors pas le principe de Hasse, le mieux qu'on puisse espérer est que l'obstruction de Manin au principe de Hasse soit la seule pour ces fibres, et c'est pour cela qu'obtenir des points adéliques de $X_{\theta}$ orthogonaux à $B_{\theta}$ (et pas seulement à la restriction de $\operatorname{Br} X$ à $X_{\theta}$ ) est crucial pour les applications. On trouvera des exemples de cette situation dans [Har94, §5]. Un cas typique où cela se produit est celui d'une hypersurface cubique lisse de dimension 3 vue comme fibrée en surfaces cubiques au-dessus de la droite projective.

4. Comme $X$ est lisse, sa fibre générique $X_{\eta}$ l'est également donc on peut supposer en plus $X_{\theta}$ lisse dans la conclusion du théorème.

5. De façon surprenante, le théorème 1 est très nettement plus difficile à démontrer que le théorème 3.2.1 de [Har97] (qui correspond grosso modo à une situation où on a pris $B=\operatorname{Br} X_{\eta}$ ) et nécessite des idées nouvelles. Ceci vient du fait qu'on a juste une hypothèse liée à une partie de $\operatorname{Br} X_{\eta}$, ce qui rend nettement moins commode la mise en place d'une récurrence sur $n$.

6. En pratique on cherche souvent à obtenir la conclusion du théorème pour un sous-groupe $C$ fini de $\operatorname{Br} X_{\eta}$ à la place de $B$. On applique alors le résultat à $B:=C+\mathrm{Br} K$; il est important de noter que l'hypothèse $X\left(\mathbf{A}_{k}\right)^{B^{\prime}} \neq \emptyset$ doit alors être vérifiée pour $B^{\prime}=B \cap \operatorname{Br} X$; la condition $X\left(\mathbf{A}_{k}\right)^{C \cap B r X} \neq \emptyset$ n'est pas suffisante.

La preuve du théorème 1 comporte deux étapes essentielles : le cas $n=1$ (pour lequel nous démontrerons un énoncé légèrement plus général), et le passage à $n$ quelconque par récurrence.

\section{Le $\operatorname{cas} n=1$}

Dans cette section, on va démontrer le suivant.

ThÉORÈme 2. Soient $k$ un corps de nombres et $X$ une $k$-variété projective, lisse et géométriquement intègre. On suppose $X$ équipée d'un morphisme $p: X \rightarrow \mathbf{P}_{k}^{1}$ projectif et surjectif dont la fibre générique $X_{\eta}$ est géométriquement intègre sur le corps des fonctions $K$ de $\mathbf{P}_{k}^{1}$. Soient alors $B$ un sous-groupe de $\operatorname{Br} X_{\eta}$ contenant $\operatorname{Br} K$ et $B^{\prime}:=B \cap \operatorname{Br} X$. On fait les hypothèses suivantes.

(a) Les fibres de $p$ au-dessus de $\mathbf{A}_{k}^{1}$ sont toutes scindées.

(b) La fibre à l'infini $X_{\infty}$ contient une composante irréductible de multiplicité 1 .

(c) Le groupe $B / \mathrm{Br} K$ est fini.

Alors si $X\left(\mathbf{A}_{k}\right)^{B^{\prime}} \neq \emptyset$, il existe pour tout sous-ensemble hilbertien $H$ de $\mathbf{P}_{k}^{1}(k)$ un point rationnel $\theta \in H$ tel que

$$
X_{\theta}\left(\mathbf{A}_{k}\right)^{B_{\theta}} \neq \emptyset \text {. }
$$

De plus si $\left(Q_{v}\right) \in X\left(A_{k}\right)^{B^{\prime}}$ et $S_{1}$ est un ensemble fini de places de $k$, alors on peut choisir $\theta$ tel que $X_{\theta}\left(\mathbf{A}_{k}\right)^{B_{\theta}}$ contienne des points $\left(P_{v}\right)$ avec $P_{v}$ arbitrairement proche de $Q_{v}$ pour $v \in S_{1}$.

La preuve du théorème 2 comporte deux ingrédients essentiels : le lemme formel (voir [Har94, corollaire 2.6.1] et [Col03, théorème 1.4]) et la proposition suivante, qui permet en quelque sorte de contrôler la ramification des éléments de $B$ à partir d'éléments de $\operatorname{Br} K$. Cette proposition est une légère généralisation du lemme 4.1.1 de [Har94]. La condition (b) du théorème 2 y remplace la condition '(Sect)' de [Har94] d'existence d'une $\bar{k}$-section générique.

Proposition 1. Soient $k$ un corps de nombres et $X$ une $k$-variété projective, lisse et géométriquement intègre, munie d'un morphisme $p: X \rightarrow \mathbf{P}_{k}^{1}$ projectif et surjectif dont la fibre 


\section{SpÉCiAlisation DES CONDitions DE MANin}

générique $X_{\eta}$ est géométriquement intègre sur le corps des fonctions $K$ de $\mathbf{P}_{k}^{1}$. Soient $B$ un sousgroupe de $\operatorname{Br} X_{\eta}$ contenant $\operatorname{Br} K$ et $B^{\prime}:=B \cap \operatorname{Br} X$. On suppose que les hypothèses (a)-(c) du théorème 2 sont satisfaites. Alors ce qui suit existe.

(1) Deux groupes finis $\Gamma \subset B$ et $E \subset \operatorname{Br} K$ tels que $: \Gamma+\operatorname{Br} K=B$ et le sous-groupe fini $(\Gamma+E)$ de $\operatorname{Br} X_{\eta}$ contient $B^{\prime}$ modulo $\operatorname{Br} k$ (i.e. $\Gamma+E+\operatorname{Br} k \supset B^{\prime}$ ).

(2) Un ouvert non vide $U_{0}$ de $\mathbf{A}_{k}^{1}$, avec $p$ lisse au-dessus de $U_{0}$, tel que tous les éléments de $\Gamma+E$ soient dans $\operatorname{Br} U$, où $U:=p^{-1}\left(U_{0}\right)$.

(3) Un ensemble infini $\Sigma$ de places de $k$ et un ensemble fini $S_{0}$ de places de $k$ (avec $S_{0}$ contenant les places archimédiennes) tels que

pour tout ensemble fini de places $S \supset S_{0}$, on ait le fait suivant.

(4) Il existe des places deux à deux distinctes $\left\{v_{1}, \ldots, v_{l}, v_{\infty}\right\}$ non dans $S$ avec $v_{\infty} \in \Sigma$, et des éléments $\theta_{i} \in \mathcal{O}_{v_{i}}$ pour $1 \leqslant i \leqslant l, \theta_{\infty} \in k_{v_{\infty}}^{*}$

qui ont la propriété suivante :

Si $\theta \in U_{0}(k)$ est un $(S \cup \Sigma)$-entier vérifiant $v_{i}\left(\theta-\theta_{i}\right) \geqslant 2$ pour $i=\{1, \ldots, l, \infty\}$ et si la fibre $X_{\theta}$ a des points locaux $M_{v}$ pour $v \in S$ vérifiant $\sum_{v \in S} j_{v}\left(\alpha\left(M_{v}\right)\right)=0$ pour tout $\alpha \in(\Gamma+E)$, alors $X_{\theta}$ possède un point adélique $\left(P_{v}\right)_{v \in \Omega_{k}}$ vérifiant $: P_{v}=M_{v}$ pour $v \in S$ et

$$
\sum_{v \in \Omega_{k}} j_{v}\left(\alpha\left(P_{v}\right)\right)=0
$$

pour tout $\alpha \in B$. En particulier $X_{\theta}\left(\mathbf{A}_{k}\right)^{B_{\theta}}$ est non vide.

Remarques. Nous faisons les remarques suivantes.

1. Si $\theta \in U_{0}$, la spécialisation de $\alpha$ dans $\operatorname{Br} X_{\theta} / \operatorname{Br} k$ a un sens pour tout $\alpha \in B$ (respectivement $\alpha \in B / \operatorname{Br} K)$ car les éléments de $\Gamma$ sont dans $\operatorname{Br} U$ et $\Gamma+\operatorname{Br} K=B$. De ce fait, la somme $\sum_{v \in \Omega_{k}} j_{v}\left(\alpha\left(P_{v}\right)\right)$ a a priori un sens pour tout $\alpha \in B$ et tout $\left(P_{v}\right) \in X_{\theta}\left(\mathbf{A}_{k}\right)$ quand $\theta \in U_{0}$.

2. L'ensemble $E \subset \operatorname{Br} K$ est celui qui, comme annoncé plus haut, permet de contrôler la ramification des éléments de $B$. Son rôle apparaîtra dans le lemme 4 .

La démonstration de la proposition 1 étant assez longue, nous la diviserons en trois étapes qui font l'objet des paragraphes suivants.

\subsection{Première étape : choix des ensembles}

2.1.1 Choix de $\Gamma$ et des points de ramification. Soit $m$ un point fermé quelconque de $\mathbf{A}_{k}^{1}$. Par hypothèse la fibre $X_{m}$ est scindée, c'est-à-dire qu'elle possède un ouvert non vide lisse $W_{m}$, de corps des fonctions $K_{m}$, qui est géométriquement intègre sur le corps résiduel $k(m)$ de $m$. On commence par choisir un système fini de représentants dans $B$ de $B / \operatorname{Br} K$ et on appelle $\Gamma$ le sous-groupe qu'ils engendrent (fini puisque $\mathrm{Br}(k(X))$ est un groupe de torsion). On peut alors trouver un ensemble fini $\left\{m_{1}, \ldots, m_{l}\right\}$ de points fermés de $\mathbf{A}_{k}^{1}$ tel que tous les éléments de $\Gamma$ soient non ramifiés en dehors de la réunion des fibres $X_{m_{1}}, \ldots, X_{m_{l}}, X_{\infty}$. On peut également supposer que les fibres de $p$ en dehors des $m_{i}$ et de $\infty$ sont lisses et géométriquement intègres. Pour simplifier l'écriture, nous noterons dans la suite $W_{i}$ pour $W_{m_{i}}$. Soient $k_{i}=k\left(m_{i}\right)$ le corps résiduel de $m_{i}$ et $K_{i}=k_{i}\left(W_{i}\right)$ le corps des fonctions de $W_{i}$. On note également $K_{i}^{\prime}$ une extension finie abélienne de $K_{i}$ telle que tous les résidus des éléments de $\Gamma$ au point générique de $W_{i}$ soient dans $H^{1}\left(\mathrm{Gal}\left(K_{i}^{\prime} / K_{i}\right), \mathbf{Q} / \mathbf{Z}\right) \subset H^{1}\left(K_{i}, \mathbf{Q} / \mathbf{Z}\right)$, puis $k_{i}^{\prime}$ la fermeture algébrique de $k_{i}$ dans $K_{i}^{\prime}$ ( $k_{i}^{\prime}$ est une extension finie abélienne de $\left.k_{i}\right)$.

2.1.2 Choix de $E$. On appelle $E_{1}$ le sous-groupe de $\operatorname{Br} K$ constitué des éléments qui sont non ramifiés en dehors de $\infty$ et des $m_{i}$, et dont les résidus en chaque $m_{i}$ sont dans $H^{1}\left(\operatorname{Gal}\left(k_{i}^{\prime} / k_{i}\right), \mathbf{Q} / \mathbf{Z}\right)$. 


\section{HARARI}

D'après la suite exacte de Faddeev (voir [Ser94, annexe au chapitre II, 2])

$$
0 \rightarrow \operatorname{Br} k \rightarrow \operatorname{Br} K \stackrel{\partial}{\rightarrow} \bigoplus_{m \in\left(\mathbf{A}_{k}^{1}\right)^{(1)}} H^{1}(k(m), \mathbf{Q} / \mathbf{Z}) \rightarrow 0
$$

(où la flèche $\partial$ est donnée par les différents résidus), le groupe $E_{1} / \mathrm{Br} k$ est fini, et on peut trouver un sous-groupe fini $E$ de $E_{1}$ qui se surjecte sur $E_{1} / \mathrm{Br} k$. On a alors le lemme suivant.

Lemme 2. Le groupe $\Gamma+E$ contient $B^{\prime}$ modulo $\operatorname{Br} k$.

Démonstration. Soit $\delta$ dans $B^{\prime}=B \cap \operatorname{Br} X$, Comme $\Gamma$ se surjecte sur $B / \operatorname{Br} K$ on peut écrire $\delta=\alpha+\beta$ avec $\alpha \in \Gamma$ et $\beta \in \operatorname{Br} K$. Soit $m$ un point fermé de $\mathbf{A}_{k}^{1}$; comme $W_{m}$ est géométriquement intègre sur $k(m)$, l'application $H^{1}(k(m), \mathbf{Q} / \mathbf{Z}) \rightarrow H^{1}\left(K_{m}, \mathbf{Q} / \mathbf{Z}\right)$ est injective et on peut identifier $H^{1}(k(m), \mathbf{Q} / \mathbf{Z})$ avec son image dans $H^{1}\left(K_{m}, \mathbf{Q} / \mathbf{Z}\right)$. Soit $\partial_{m}(\alpha)$ (respectivement $\partial_{m}(\beta)$ ) le résidu de $\alpha$ au point générique de $W_{m}$ (respectivement de $\beta$ en $m$ ). Comme $\alpha+\beta$ est non ramifié sur $X$ tout entier, on a $\partial_{m}(\alpha)+\partial_{m}(\beta)=0$, donc $\partial_{m}(\beta)=0$ si $m$ n'est pas l'un des $m_{i}$. D'autre part $\partial_{m_{i}}(\alpha)$ est dans $H^{1}\left(\operatorname{Gal}\left(K_{i}^{\prime} / K_{i}\right), \mathbf{Q} / \mathbf{Z}\right)$ avec $k_{i}^{\prime}$ algébriquement clos dans $K_{i}^{\prime}$, ce qui impose que $\partial_{m_{i}}(\beta)$ (qui est déjà dans $\left.H^{1}\left(k_{i}, \mathbf{Q} / \mathbf{Z}\right)\right)$ soit dans $H^{1}\left(\mathrm{Gal}\left(k_{i}^{\prime} / k_{i}\right), \mathbf{Q} / \mathbf{Z}\right)$. Ainsi $\beta \in E_{1}=E+\operatorname{Br} k$ et finalement $\delta \in \Gamma+E+\operatorname{Br} k$, ce qui prouve que $\Gamma+E$ contient $B^{\prime}$ modulo $\operatorname{Br} k$, d'où le lemme.

On a donc (1). Posons alors $U_{0}=\mathbf{A}_{k}^{1} \backslash\left\{m_{1}, \ldots, m_{l}\right\}$, on obtient (2).

2.1.3 Choix de $\Sigma$. La fibre à l'infini contient une composante de multiplicité 1, donc un ouvert non vide $W_{\infty}$ (désormais fixé) qui est intègre. Soit $k_{\infty}$ la fermeture algébrique de $k$ dans le corps des fonctions $K_{\infty}$ de $W_{\infty}$. On appelle $K_{\infty}^{\prime}$ une extension finie abélienne de $K_{\infty}$ tels que les résidus des éléments de $\Gamma$ au point générique de $W_{\infty}$ soient dans $H^{1}\left(\mathrm{Gal}\left(K_{\infty}^{\prime} / K_{\infty}\right), \mathbf{Q} / \mathbf{Z}\right)$, puis $k_{\infty}^{\prime}$ la fermeture algébrique de $k_{\infty}$ dans $K_{\infty}^{\prime}$. Comme $\operatorname{Br}(\bar{k}(T))=0$ par le théorème de Tsen, il existe une extension finie galoisienne $L / k$ telle que la restriction à $\operatorname{Br}(L(T))$ de tous les éléments de $E$ soit nulle. On choisit alors pour $\Sigma$ un ensemble infini de places qui sont totalement décomposées dans $k_{\infty}^{\prime} / k$, et également dans $L / k$ (ceci existe d'après le théorème de Chebotarev). En particulier si $w$ est une place de $\Sigma$, les éléments de $E$ ont tous une image nulle dans $\operatorname{Br}\left(k_{w}(T)\right)$. Quitte à restreindre les ouverts $W_{1}, \ldots, W_{l}, W_{\infty}$, on peut également supposer que les résidus des éléments de $\Gamma+E$ au point générique de $W_{i}$ sont dans $H^{1}\left(W_{i}^{\prime} / W_{i}, \mathbf{Q} / \mathbf{Z}\right)$, où $W_{i}^{\prime}$ est un revêtement étale connexe de $W_{i}$ de corps des fonctions $K_{i}^{\prime}$ pour $i=1, \ldots, l, \infty$.

2.1.4 Choix de $S_{0}$. Il reste à choisir $S_{0}$ comme dans (3). Soit $P_{i} \in k[T]$ unitaire tel que $k_{i}=$ $k[T] / P_{i}$ pour $1 \leqslant i \leqslant l$. On commence par prendre $S_{0}$ tel que pour $v \notin S_{0}$, les polynômes $P_{i}$ $(1 \leqslant i \leqslant l)$ soient à coefficients dans $\mathcal{O}_{v}$, avec leurs réductions modulo $v$ séparables et premières entre elles deux à deux. On demande ensuite que $X$ s'étende en un schéma propre et lisse $\mathcal{X}$ audessus de $\operatorname{Spec} \mathcal{O}_{k, S_{0}}$; on appelle alors $\mathcal{U}_{0}$ le complémentaire de l'adhérence de Zariski de l'ensemble des $m_{i}$ dans $\mathbf{A}_{\mathcal{O}_{k, S_{0}}}^{1}$ et on pose $\mathcal{U}=p^{-1}\left(\mathcal{U}_{0}\right)$. On peut aussi supposer que les éléments de $\Gamma+E$ sont dans $\operatorname{Br} \mathcal{U}$ et que les $W_{i}$ s'étendent en des $\mathcal{W}_{i}$ lisses sur $\mathcal{O}_{k, S_{0}}$ pour $i=1, \ldots, l, \infty$. On prend aussi $S_{0}$ tel que les revêtements étales $W_{i}^{\prime} / W_{i}$ s'étendent en des revêtements étales $\mathcal{W}_{i}^{\prime} / \mathcal{W}_{i}$ au-dessus de $\mathcal{O}_{k, S_{0}}$, avec les résidus des éléments de $\Gamma+E$ au point générique de $W_{i}$ dans $H^{1}\left(\mathcal{W}_{i}^{\prime} / \mathcal{W}_{i}, \mathbf{Q} / \mathbf{Z}\right)$.

Si $\mathcal{Z}$ est un $\mathcal{O}_{v}$-schéma, notons $\widetilde{Z}$ sa réduction modulo $v$. Par le théorème de Lang-Weil on peut (quitte à agrandir encore $S_{0}$ ) supposer que pour toute place $v \notin S_{0}$ : la fibre de $\widetilde{U}$ au-dessus de tout $k(v)$-point de $\widetilde{U}_{0}$ possède un $k(v)$-point.

Si $i=1, \ldots, l$, la fibre de $\widetilde{W}_{i}$ au-dessus de tout $k(v)$-point de $\tilde{m}_{i}$ possède un $k(v)$-point; bien sûr il y a des places $v$ pour lesquelles $\tilde{m}_{i}$ n'aura pas de $k(v)$-point du tout, auquel cas ce dernier 


\section{SpÉCiAlisation DES CONDitions DE MANin}

enoncé est vide, mais cette formulation est commode pour éviter de travailler avec les places de $k_{i}$. On utilisera ceci quand $v$ est (partiellement) scindée dans $k_{i} / k$, donc quand $\tilde{m}_{i}$ a bien un $k(v)$-point.

Si $v$ est totalement décomposée dans $k_{\infty} / k, \widetilde{W}_{\infty}$ possède un $k(v)$-point.

Et enfin d'après le lemme 1.2. de [Eke90], on peut aussi imposer : si $i \in\{1, \ldots, l, \infty\}$ et si de plus $v$ est totalement décomposée dans $k_{i}^{\prime} / k$, alors il existe pour tout $\sigma$ de $\operatorname{Gal}\left(K_{i}^{\prime} / k_{i}^{\prime} K_{i}\right)$ un $k(v)$-point $\widetilde{P}_{v}$ de $\widetilde{W}_{i}$ dont le Frobenius associé (pour le revêtement $\mathcal{W}_{i}^{\prime} / \mathcal{W}_{i}$ ) est $\sigma$.

Nous appellerons par la suite 'propriété (F)' l'existence de ces différents points sur les corps finis.

2.1.5 Choix des $v_{i}$ et $\theta_{i}$. Soit alors $S \supset S_{0}$ un ensemble fini arbitraire de places de $k$. On choisit des places $v_{1}, \ldots, v_{l}$ deux à deux distinctes de $k$, non dans $S$, avec $v_{i}$ totalement décomposée dans $k_{i}^{\prime} / k$, puis $v_{\infty}$ dans $\Sigma$ avec $v_{\infty} \notin S \cup\left\{v_{1}, \ldots, v_{l}\right\}$. On fixe aussi $\theta_{i} \in \mathcal{O}_{v_{i}}$ avec $v_{i}\left(P_{i}\left(\theta_{i}\right)\right)=1$ pour $1 \leqslant i \leqslant l$; ceci est possible via le lemme de Hensel parce que les polynômes $P_{i}$ sont séparables modulo $v_{i}$ et possèdent une racine dans $k\left(v_{i}\right)$ vu que $v_{i}$ est totalement décomposée dans $k_{i}^{\prime} / k$, donc a fortiori dans $k_{i} / k$. On choisit enfin $\theta_{\infty} \in k_{v_{\infty}}^{*}$ avec $v_{\infty}\left(1 / \theta_{\infty}\right)=1$ pour obtenir (4).

\subsection{Deuxième étape : existence de points adéliques $\left(M_{v}\right)$ dans les fibres et calcul de $j_{v}\left(\alpha\left(M_{v}\right)\right)$}

Fixons un $(S \cup \Sigma)$-entier $\theta \in U_{0}(k)$ vérifiant $v_{i}\left(\theta-\theta_{i}\right) \geqslant 2$ pour $i=1, \ldots, l, \infty$. En particulier $v_{i}\left(P_{i}(\theta)\right)=1$ pour $1 \leqslant i \leqslant l$ et $v_{\infty}(\theta)=-1$.

Montrons un lemme.

Lemme 3. La fibre $X_{\theta}$ possède un $k_{v}$-point $M_{v}$ pour toute place $v$. De plus on peut demander que pour $v$ non dans $S$, la réduction de $M_{v}$ soit dans $\widetilde{W}_{i}$ si $v\left(P_{i}(\theta)\right)>0$, dans $\widetilde{W}_{\infty}$ si $v(\theta)<0$, et dans $\mathcal{U}$ sinon.

Démonstration. Par hypothèse, des points $M_{v}$ sur $X_{\theta}$ (vérifiant la formule $\sum_{v \in S} j_{v}\left(\alpha\left(M_{v}\right)\right)=0$ pour tout $\alpha \in \Gamma+E$ ) nous sont donnés. Pour $v \notin S$, on va utiliser le lemme de Hensel et le fait que $S_{0}$ a été choisi pour vérifier la propriété $(\mathrm{F})$. Les trois cas suivant sont possibles si $v \notin S$.

(i) La réduction modulo $v$ de $\theta$ est dans $\widetilde{U}_{0}$, i.e. $v(\theta) \geqslant 0$ et $v\left(P_{i}(\theta)\right)=0$ pour $1 \leqslant i \leqslant l$. On obtient alors $M_{v}$ en relevant un $k(v)$-point de $\widetilde{U}$.

(ii) La réduction modulo $v$ de $\theta$ est à l'infini, i.e. $v(\theta)<0$. Alors $v \in \Sigma$ car $\theta$ est un $(S \cup \Sigma)$ entier. Mais alors $\widetilde{W}_{\infty}$ possède un $k(v)$-point (car $v$ est totalement décomposée dans $k_{\infty} / k$ donc $k_{\infty}(w)=k(v)$ pour toute place $w$ de $\left.k_{\infty}\right)$ qu'on relève en $M_{v} \in X_{\theta}\left(k_{v}\right)$.

(iii) La réduction modulo $v$ de $\theta$ est 'l'un des $m_{i}$ ', plus précisément $v\left(P_{i}(\theta)\right)>0$ pour un certain $i$ de $1, \ldots, l$ (nécessairement unique car les $P_{i}$ modulo $v$ sont deux à deux premiers entre eux). Ceci implique qu'il existe une place $w$ de $k_{i}$ au-dessus de $v$ avec $k_{i}(w)=k(v)$, et on obtient $M_{v}$ en relevant un $k_{i}(w)$-point de $\widetilde{W}_{i}$.

Finalement on obtient bien que $X_{\theta}$ possède un point adélique satisfaisant les conditions voulues.

On fixe désormais un point adélique $\left(M_{v}\right)$ de $X_{\theta}$ avec $\sum_{v \in S} j_{v}\left(\alpha\left(M_{v}\right)\right)=0$ pour tout $\alpha \in \Gamma+E$. Dans le calcul de $j_{v}\left(\alpha\left(M_{v}\right)\right)$ quand $v \notin S$, on retrouve les trois cas ci-dessus pour $v$.

(i) La réduction modulo $v$ de $\theta$ est dans $\widetilde{U}_{0}$, alors $M_{v} \in \mathcal{U}\left(\mathcal{O}_{v}\right)$ donc $\alpha\left(M_{v}\right)=0$ puisque $(\Gamma+E) \subset$ Br $\mathcal{U}$. On appelle $\Omega_{0}$ l'ensemble de ces places.

(ii) Nous avons $v(\theta)<0$, i.e. la réduction $\widetilde{M}_{v}$ de $M_{v}$ est dans $\widetilde{W}_{\infty}$. Alors par une formule bien connue [Har94, corollaire 2.4.3 et pp. 244-245] on a

$$
j_{v}\left(\alpha\left(M_{v}\right)\right)=n_{\infty}(v) \partial_{\alpha, \infty}\left(F_{\infty, \widetilde{M}_{v}}\right)
$$




\section{HARARI}

où:

(a) $n_{\infty}(v)=v(1 / \theta)$ est la multiplicité (qui est $\left.>0\right)$;

(b) $\partial_{\alpha, \infty} \in H^{1}\left(\operatorname{Gal}\left(K_{\infty}^{\prime} / K_{\infty}\right), \mathbf{Q} / \mathbf{Z}\right)$ est le résidu de $\alpha$ au point générique de $W_{\infty}$;

(c) $F_{\infty, \widetilde{M}_{v}} \in \mathrm{Gal}\left(K_{\infty}^{\prime} / K_{\infty}\right)$ est le Frobenius en $\widetilde{M}_{v}$ associé au revêtement $\mathcal{W}_{\infty}^{\prime} / \mathcal{W}_{\infty}$.

On appelle $\Omega_{\infty}$ l'ensemble de ces places (qui est fini, $\theta$ étant fixé); notons que $\Omega_{\infty} \subset \Sigma$ et $v_{\infty} \in \Omega_{\infty}$.

(iii) Nous avons $v\left(P_{i}(\theta)\right)>0$ pour un certain $i$, i.e. la réduction $\widetilde{M}_{v}$ de $M_{v}$ est dans $\widetilde{W}_{i}$. La formule est similaire :

$$
j_{v}\left(\alpha\left(M_{v}\right)\right)=n_{i}(v) \partial_{\alpha, i}\left(F_{i, \widetilde{M}_{v}}\right)
$$

où $n_{i}(v)=v\left(P_{i}(\theta)\right)>0, \partial_{\alpha, i}$ est le résidu de $\alpha$ au point générique de $W_{i}$ et $F_{i, \widetilde{M}_{v}}$ est le Frobenius en $\widetilde{M}_{v}$ associé au revêtement $\mathcal{W}_{i}^{\prime} / \mathcal{W}_{i}$. On appelle $\Omega_{i}$ l'ensemble de ces places pour chaque $i=1, \ldots, l$, qui est également fini et contient $v_{i}$.

\subsection{Troisième étape : construction des points $\boldsymbol{P}_{\boldsymbol{v}}$ et fin de la preuve de la proposition 1}

Si $v \in S$ on prend pour $P_{v}$ le point $M_{v}$ fixé plus haut (qui avait été fourni par l'énoncé de la proposition 1). Si $v$ n'est ni dans $S$ ni dans $\left\{v_{1}, \ldots, v_{l}, v_{\infty}\right\}$, on prend également $P_{v}=M_{v}$ (ici $M_{v}$ avait été donné par le lemme 3). Quand $v \in\left\{v_{1}, \ldots, v_{l}, v_{\infty}\right\}$, nous allons modifier les points $M_{v}$ donnés par ce même lemme, de sorte que le nouveau point adélique de $X_{\theta}$ ainsi obtenu soit dans $X_{\theta}\left(\mathbf{A}_{k}\right)^{B_{\theta}}$. Nous utiliserons le lemme suivant.

Lemme 4. Soit $i \in\{1, \ldots, l, \infty\}$. Alors l'élément

$$
\sum_{v \in \Omega_{i}} n_{i}(v) F_{i, \widetilde{M}_{v}}
$$

de $\operatorname{Gal}\left(K_{i}^{\prime} / K_{i}\right)$ appartient au sous-groupe $\operatorname{Gal}\left(K_{i}^{\prime} / k_{i}^{\prime} K_{i}\right)$.

On va voir que la condition voulue est forcée par la condition que l'on a imposée sur $E$, à savoir $\sum_{v \in S} j_{v}\left(\alpha\left(M_{v}\right)\right)=0$ pour tout $\alpha$ de $E$.

Démonstration. Pour $i=\infty$, c'est clair car tout $v \in \Omega_{\infty}$ est dans $\Sigma$ donc est totalement décomposée pour $k_{\infty}^{\prime} / k_{\infty}$, ce qui fait que le Frobenius $F_{\infty, \widetilde{M}_{v}}$ est automatiquement dans $\operatorname{Gal}\left(K_{\infty}^{\prime} / k_{\infty}^{\prime} K_{\infty}\right)$. Soit $i \in\{1, \ldots, l\}$ fixé et $\rho_{i}$ arbitraire dans $H^{1}\left(\operatorname{Gal}\left(k_{i}^{\prime} / k_{i}\right), \mathbf{Q} / \mathbf{Z}\right)=H^{1}\left(\mathrm{Gal}\left(k_{i}^{\prime} K_{i} / K_{i}\right), \mathbf{Q} / \mathbf{Z}\right)$. D'après la suite exacte de Faddeev, il existe $A_{i}$ dans $\operatorname{Br} K$ dont le seul résidu sur $\mathbf{A}_{k}^{1}$ est en $m_{i}$ et vaut $\rho_{i}$; par définition de $E_{1}$, on a $A_{i} \in E_{1}$ et comme $E_{1}=E+\operatorname{Br} k$, on peut supposer que $A_{i} \in E$. La spécialisation de $A_{i}$ en $X_{\theta}$ étant dans $\operatorname{Br} k$, on a

$$
\sum_{v \in \Omega_{k}} j_{v}\left(A_{i}\left(M_{v}\right)\right)=0
$$

D'autre part on avait $\sum_{v \in S} j_{v}\left(A_{i}\left(M_{v}\right)\right)=0$ puisque $A_{i}$ est dans $E$. Si $v$ n'est pas dans $S \cup \Omega_{\infty} \cup \Omega_{i}$, on a $A_{i}\left(M_{v}\right)=0$ d'après le calcul de $\alpha\left(M_{v}\right)$ (pour $\alpha \in \Gamma+E$ ) fait plus haut, car $A_{i}$ est dans $\operatorname{Br} \mathcal{U}_{0}$ et non ramifiée en dehors de $m_{i}$ et $\infty$.

D'autre part $A_{i}\left(M_{w}\right)=0$ pour toute place $w$ de $\Omega_{\infty} \subset \Sigma$ car $A_{i}$ est alors tué dans $\operatorname{Br}\left(k_{w}(T)\right)$. On en déduit finalement

ce qui se traduit, vu la formule (1) par

$$
\sum_{v \in \Omega_{i}} j_{v}\left(A_{i}\left(M_{v}\right)\right)=0
$$

$$
\rho_{i}\left(\sum_{v \in \Omega_{i}} n_{i}(v) F_{i, \widetilde{M}_{v}}\right)=0 .
$$

Comme $\rho_{i}$ était arbitraire dans $H^{1}\left(\operatorname{Gal}\left(k_{i}^{\prime} K_{i} / K_{i}\right), \mathbf{Q} / \mathbf{Z}\right)$, la preuve du lemme est achevée. 


\section{Spécialisation Des CONDitions DE MANin}

On peut maintenant terminer la preuve de la proposition 1 . Pour chaque $i \in\{1, \ldots, l, \infty\}$, on peut (d'après le dernier point de la propriété $(\mathrm{F})$ ) trouver un point $\widetilde{P}_{v_{i}}$ dans $\widetilde{W}_{i}$ dont le Frobenius associé $F_{i, \widetilde{P}_{v_{i}}}$ est $F_{i, \widetilde{M}_{v_{i}}}-\sum_{v \in \Omega_{i}} n_{i}(v) F_{i, \widetilde{M}_{v}}$. En effet $v_{i}$ est totalement décomposée dans $k_{i}^{\prime} / k$ et $F_{i, \widetilde{M}_{v_{i}}}-\sum_{v \in \Omega_{i}} n_{i}(v) F_{i, \widetilde{M}_{v}}$ est dans Gal $\left(K_{i}^{\prime} / k_{i}^{\prime} K_{i}\right)$ d'après le lemme 4 . On relève alors $\widetilde{P}_{v_{i}}$ en $P_{v_{i}} \in$ $X_{\theta}\left(k_{v_{i}}\right)$. Alors comme $n_{i}\left(v_{i}\right)=1$ vu que $v_{i}(\theta)=1$ pour $i=1, \ldots, l$ et $v_{\infty}(1 / \theta)=1$, on obtient pour tout $\alpha \in \Gamma$ :

$$
\sum_{v \in \Omega_{i}} j_{v}\left(\alpha\left(P_{v}\right)\right)=\partial_{\alpha, i}\left(\sum_{v \in \Omega_{i}} n_{i}(v) F_{i, \widetilde{P}_{v}}\right)=\partial_{\alpha, i}(0)=0 .
$$

D'autre part $P_{v}=M_{v}$ en dehors de $v_{1}, \ldots, v_{l}, v_{\infty}$ d'où $\alpha\left(P_{v}\right)=0$ si $v \in \Omega_{0}$ et $\sum_{v \in S} j_{v}\left(\alpha\left(P_{v}\right)\right)=0$. Comme $\Omega_{0}, \Omega_{1}, \ldots, \Omega_{l}, \Omega_{\infty}$ et $S$ réalisent une partition de $\Omega_{k}$, on obtient finalement

$$
\sum_{v \in \Omega_{k}} j_{v}\left(\alpha\left(P_{v}\right)\right)=0
$$

pour tout $\alpha \in \Gamma$, donc aussi pour tout $\alpha \in B$ puisque $\Gamma+\operatorname{Br} K=B$.

\subsection{Preuve du théorème 2}

La proposition 1 nous fournit des sous-groupes finis $\Gamma \subset B$ et $E \subset \operatorname{Br} K$, ainsi que des ensembles de places $\Sigma$ (infini) et $S_{0}$ (fini), puis un ouvert $U_{0}$ de $\mathbf{A}_{k}^{1}$. Soit $\left(Q_{v}\right)$ dans $X\left(\mathbf{A}_{k}\right)^{B^{\prime}}$ (qui est non vide par hypothèse). Comme $\Gamma+E$ est fini, nous pouvons appliquer le lemme formel (voir [Har94, corollaire 2.6.1] et [Col03, théorème 1.4]) : il existe $S \supset\left(S_{0} \cup S_{1}\right)$ et des points $R_{v}$ pour $v \in S$ vérifiant, $R_{v}=Q_{v}$ pour $v \in\left(S_{0} \cup S_{1}\right)$ et $\sum_{v \in S} j_{v}\left(\alpha\left(R_{v}\right)\right)=0$ pour tout $\alpha \in(\Gamma+E)$. La proposition 1 donne alors des places $v_{1}, \ldots, v_{l}, v_{\infty}$ (rappelons que $v_{\infty} \in \Sigma$ ) et des éléments $\theta_{1}, \ldots, \theta_{l}, \theta_{\infty}$ avec $\theta_{i} \in \mathcal{O}_{v_{i}}$ pour $i=1, \ldots, l$ et $\theta_{\infty} \in k_{v_{\infty}}^{*}$.

Choisissons une place $w$ de $\Sigma$ non dans $S \cup\left\{v_{1}, \ldots, v_{l}, v_{\infty}\right\}$ (c'est possible car $\Sigma$ est infini). Par le théorème d'irréductibilité de Hilbert avec approximation forte sur $\mathbf{A}_{k}^{1}$ (voir [Eke90, Theorem 1.3]; [Har94, proposition 3.2.1]), il existe $\theta$ dans $U_{0}(k) \cap H$, arbitrairement proche de $p\left(R_{v}\right)$ pour $v \in S$ et de $\theta_{i}$ pour $i=1, \ldots, l, \infty$, avec $\theta$ entier en dehors de $S \cup\left\{v_{1}, \ldots, v_{l}, v_{\infty}, w\right\}$. En particulier $\theta$ est un $(S \cup \Sigma)$-entier. Par le théorème des fonctions implicites, la fibre $X_{\theta}$ possède alors des point $M_{v}$ pour $v$ dans $S$ qui sont arbitrairement proches de $R_{v}$, donc vérifient aussi $\sum_{v \in S} j_{v}\left(\alpha\left(M_{v}\right)\right)=0$. Notons que pour $v \in S_{1}, M_{v}$ est proche du point $Q_{v}$ donné au départ car $R_{v}=Q_{v}$ pour $v \in S_{1}$. La proposition 1 dit alors que $X_{\theta}$ possède un point adélique $\left(P_{v}\right)$ avec $P_{v}=M_{v}$ pour $v \in S$ (donc $P_{v}$ est proche de $Q_{v}$ pour $\left.v \in S_{1}\right)$ avec $\left(P_{v}\right) \in X_{\theta}\left(\mathbf{A}_{k}\right)^{B_{\theta}}$, ce qui est exactement le résultat voulu.

\section{Preuve du théorème 1 pour $n$ quelconque}

Nous démontrons le résultat par récurrence $\operatorname{sur} n$. Le cas $n=1$ résulte immédiatement du théorème 2 . Soit donc $n \geqslant 2$, supposons le résultat démontré pour tout entier $<n$. Soit $p: X \rightarrow \mathbf{P}_{k}^{n}$ comme dans le théorème 1 (en particulier les fibres de $p$ sont scindées en dehors d'un fermé $F$ de $\mathbf{P}_{k}^{n}$ de codimension au moins 2).

\subsection{Construction d'une fibration au-dessus de $\mathrm{P}_{k}^{n-1}$}

L'idée est de se ramener à l'analogue d'une situation au-dessus de $\mathbf{P}_{k}^{1} \times \mathbf{P}_{k}^{n-1}$ à l'aide du lemme suivant.

Lemme 5. Il existe une $k$-variété projective, lisse, géométriquement intègre $Y$ et un fibré en droites projectives $\pi_{1}: \Delta \rightarrow \mathbf{P}_{k}^{n-1}$ vérifiant les propriétés suivantes.

(a) La variété $Y$ est munie d'un $k$-morphisme projectif birationnel $q: Y \rightarrow X$ et d'un $k$-morphisme projectif et surjectif $g: Y \rightarrow \Delta$, tels que le morphisme composé $f_{1}:=\pi_{1} \circ g: Y \rightarrow \mathbf{P}_{k}^{n-1}$ soit projectif, surjectif, et à fibres scindées. 


\section{HARARI}

(b) Il existe un $k$-morphisme projectif et birationnel $\pi_{2}: \Delta \rightarrow \mathbf{P}_{k}^{n}$ tel que le diagramme suivant soit commutatif.

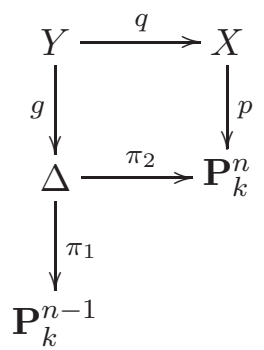

En particulier, soient $f: Y \rightarrow \mathbf{P}_{k}^{n}$ le morphisme composé $p \circ q$ et $m$ un point quelconque de $\mathbf{P}_{k}^{n-1}$; alors la fibre de $g_{m}: Y_{m} \rightarrow \Delta_{m}$ en un point $\theta_{1}$ de $\Delta_{m}$ n'est autre que la fibre de $f$ en $\theta:=\pi_{2}\left(\theta_{1}\right)$, où $g_{m}$ désigne la fibre de $g$ en $\Delta_{m}:=\pi_{1}^{-1}(m)$.

(c) Il existe un ouvert non vide $U$ de $\mathbf{P}_{k}^{n-1}$ tel que pour tout $m \in U$ de corps résiduel $k(m)$, on ait : la $k(m)$-variété $Y_{m}$ est géométriquement intègre ; le morphisme $g_{m}$ est projectif, surjectif, à fibres scindées, et à fibre générique géométriquement intègre. à $\mathbf{P}_{k(m)}^{1}$.

Notons que comme $\pi_{1}$ est un fibré en droites projectives, la fibre $\Delta_{m}$ de $\pi_{1}$ en $m$ est isomorphe

Démonstration. Rappelons tout d'abord le théorème de Bertini suivant [Jou83, corollaire 6.11.c] : pour $X$ géométriquement intègre et équipée d'un morphisme vers $\mathbf{P}_{k}^{n}$ dont l'adhérence de l'image est de dimension au moins $d+1$ (avec $d>0$ ), l'image réciproque de presque toutes les variétés linéaires de codimension $d$ de $\mathbf{P}_{k}^{n}$ est géométriquement intègre. On applique ici ce résultat avec $d=n-1$, vu que l'image de $X$ par $p$ est $\mathbf{P}_{k}^{n}$ tout entier avec $n \geqslant 2$. On en déduit qu'il existe un point $O$ de $\mathbf{P}_{k}^{n}$ tel que l'image réciproque par $p$ d'au moins une droite (donc de presque toutes les droites) passant par $O$ soit géométriquement intègre. Comme la fibre générique de $p$ est lisse et géométriquement intègre, on peut également supposer que la fibre en $O$ est lisse et géométriquement intègre. On choisit un système de coordonnées homogènes de $\mathbf{P}_{k}^{n}$ tel que $O=(0 ; 0,0, \ldots, 1)$.

Soit $\Delta$ l'éclaté de $\mathbf{P}_{k}^{n}$ en $O$, qu'on peut voir comme la sous-variété (qui est lisse) de $\mathbf{P}_{k}^{n-1} \times \mathbf{P}_{k}^{n}$ donnée par les équations $x_{i} m_{j}-x_{j} m_{i}=0$ pour $1 \leqslant i, j \leqslant n-1$, où $\left(\left(m_{0} ; m_{1}, \ldots, m_{n-1}\right)\right.$, $\left.\left(x_{0} ; x_{1}, \ldots, x_{n}\right)\right)$ sont les coordonnées homogènes d'un point $(m, x)$ de $\mathbf{P}_{k}^{n-1} \times \mathbf{P}_{k}^{n}$. Si on identifie $\mathbf{P}_{k}^{n-1}$ à l'ensemble des droites de $\mathbf{P}_{k}^{n}$ passant par $O$, cela revient à définir $\Delta$ comme l'ensemble des $(m, x)$ avec $x \in m$. La première projection $\pi_{1}: \Delta \rightarrow \mathbf{P}_{k}^{n-1}$ fait de $\Delta$ un fibré en droites projectives au-dessus de $\mathbf{P}_{k}^{n-1}$. La deuxième projection $\pi_{2}: \Delta \rightarrow \mathbf{P}_{k}^{n}$ induit un isomorphisme au-dessus de $V:=\mathbf{P}_{k}^{n}-\{O\}$. On définit alors $Y$ par $Y:=X \times \mathbf{P}_{k}^{n} \Delta$.

Montrons que $Y$ est une $k$-variété lisse. Les images réciproques $Y_{V}, X_{V}$ de $V$ dans $Y, X$ sont isomorphes (donc lisses car $X$ est lisse) ; comme la fibre de $X$ en $O$ est lisse, il existe un ouvert $W$ de $\mathbf{P}_{k}^{n}$ contenant $O$ tel que la restriction de $p$ à $p^{-1}(W) \subset X$ soit un morphisme lisse ; ainsi la restriction de $g: Y \rightarrow \Delta$ à l'image réciproque $Y_{W}$ de $\pi_{2}^{-1}(W) \subset \Delta$ est également lisse, d'où la lissité de $Y_{W}$ vu que $\pi_{2}^{-1}(W)$ est un ouvert de $\Delta$ (qui est lisse). Comme $Y$ est la réunion des ouverts $Y_{V}$ et $Y_{W}$, la lissité de $Y$ en découle.

La première projection $q: Y \rightarrow X$ est un morphisme birationnel car $\pi_{2}$ était déjà birationnelle. Soit $g: Y \rightarrow \Delta$ la deuxième projection. Par construction, on a $\pi_{2} \circ g=p \circ q$, d'où (b).

Montrons que pour tout $m \in \mathbf{P}_{k}^{n-1}$, la $k(m)$-variété $Y_{m}$ est scindée. C'est l'image réciproque de la droite projective $\Delta_{m}$ par $p: X \rightarrow \mathbf{P}_{k}^{n}$. Elle est munie d'un morphisme surjectif $g_{m}$ vers $\Delta_{m} \simeq \mathbf{P}_{k(m)}^{1}$ dont la fibre générique $Y_{\eta_{m}}$ est lisse et géométriquement intègre (la fibre en $O$ l'est). L'une des composantes irréductibles de $Y_{m}$ domine $\Delta_{m}$, et contient un ouvert non vide lisse $\omega$ qui 


\section{SpÉCiAlisation DES CONDitions DE MANin}

est intègre (car irréductible et réduit). Comme le corps des fonctions de $\omega$ est celui de $Y_{\eta_{m}}$, il ne peut contenir d'extension finie de $k(m)$. D'où (a).

Soit alors $U$ l'ouvert de $\mathbf{P}_{k}^{n-1}$ obtenu en enlevant 'les droites passant par $O$ qui rencontrent $F$ '. Plus précisément, on pose $U=\mathbf{P}_{k}^{n-1} \backslash F_{1}$, où $F_{1}$ est la projection dans $\mathbf{P}_{k}^{n-1}$ (via $\pi_{1}$ ) de l'image réciproque (via $\pi_{2}$ ) de $F$ dans $\Delta$, laquelle est isomorphe à $F$ puisque $O \notin F$. On a bien $F_{1} \neq \mathbf{P}_{k}^{n-1}$ car $F$ était de codimension au moins 2 dans $\mathbf{P}_{k}^{n}$. D'après le choix de $O$ fait au début, on peut supposer (quitte à restreindre $U$ ) que pour $m \in U$, la $k(m)$-variété $Y_{m}=p^{-1}\left(\Delta_{m}\right)$ est géométriquement intègre. Pour $m \in U$, les fibres de $g_{m}: Y_{m} \rightarrow \Delta_{m}$ sont scindées puisque la droite $\Delta_{m}$ ne rencontre pas $F$, et on a déjà vu que la fibre générique de $g_{m}$ était géométriquement intègre. D'où (c).

\subsection{Utilisation de l'hypothèse de récurrence}

Nous reprenons la preuve du théorème 1. La stratégie est la suivante : d'abord grâce au lemme 5 , on se ramène à une variété $Y$ fibrée au-dessus de $\mathbf{P}_{k}^{1} \times \mathbf{P}_{k}^{n-1}$. Les $k$-fibres du morphisme $f_{1}: Y \rightarrow \mathbf{P}_{k}^{n-1}$ correspondent à l'image réciproque des droites de $\mathbf{P}_{k}^{n}$ par le morphisme initial $p$; la proposition 2 va permettre d'appliquer à chacune de ces fibres $f_{m}: Y_{m} \rightarrow \mathbf{P}_{k}^{1}$ le cas $n=1$ du théorème 1 avec un sous-groupe 'contrôlable' du groupe de Brauer de la fibre générique de $f_{m}$. On en déduit que les $k$-fibres de $f_{1}$ satisfont bien les conditions nécessaires pour appliquer l'hypothèse de récurrence. L'analogue de la condition de finitude de $B / \operatorname{Br} K$ (dans le théorème 1 ) au rang $n-1$ sera assurée par le lemme 6.

Soit donc $Y$ comme dans le lemme 5 et $f: Y \rightarrow \mathbf{P}_{k}^{n}$ la composée de $q$ et $p$. Vu l'invariance birationnelle du groupe de Brauer, il nous suffit de démontrer le théorème pour $f$ au lieu de $p$ vu que $q: Y \rightarrow X$ est une application birationnelle et qu'il existe un ouvert non vide $V^{\prime}$ de $\mathbf{P}_{k}^{n}$ tel que pour tout $u$ de $V^{\prime}$, les fibres de $Y$ et $X$ soient projectives, lisses et birationnelles sur le corps résiduel de $u$.

Soient $B$ un sous-groupe de $\operatorname{Br} Y_{\eta}$ contenant $\operatorname{Br} K$, avec $B / \operatorname{Br} K$ fini, et $B^{\prime}:=B \cap \operatorname{Br} Y$. Ici $\eta$ désigne le point générique de $\mathbf{P}_{k}^{n}$ et $K$ son corps des fonctions. Supposons $Y\left(\mathbf{A}_{k}\right)^{B^{\prime}}$ non vide. Soient $H$ un sous-ensemble hilbertien de $\mathbf{P}_{k}^{n}(k)$ et $\left(M_{v}\right) \in Y\left(\mathbf{A}_{k}\right)^{B^{\prime}}$, puis $S_{1}$ un ensemble fini de places de $k$. On veut démontrer que $H$ contient un point rationnel $\theta$ tel que $Y_{\theta}\left(\mathbf{A}_{k}\right)^{B_{\theta}}$ possède un point $\left(P_{v}\right)$ avec $\left(P_{v}\right)$ arbitrairement proche de $M_{v}$ pour $v$ dans $S_{1}$.

Pour cela, on commence par regarder la fibre de $g: Y \rightarrow \Delta$ au-dessus de $\Delta_{\eta_{1}} \simeq \mathbf{P}_{K_{1}}^{1}$, où $\eta_{1}$ est le point générique de $\mathbf{P}_{k}^{n-1}$ et $K_{1}$ son corps des fonctions. On obtient un morphisme projectif et surjectif $g_{1}: Y_{\eta_{1}} \rightarrow \Delta_{\eta_{1}}$. Notons que les éléments de $B$ sont non ramifiés sur la fibre générique de $g_{1}$ (qui n'est autre que $Y_{\eta}$ ). Posons alors $C:=B \cap \operatorname{Br} Y_{\eta_{1}}$. Le corps des fonctions $K_{1}\left(\Delta_{\eta_{1}}\right)$ de $\Delta_{\eta_{1}}$ est $K$.

Lemme 6. Le groupe $C$ est fini modulo $\mathrm{Br} K_{1}$.

Démonstration. On va d'abord montrer que l'intersection de $\operatorname{Br} K=\operatorname{Br}\left(K_{1}\left(\Delta_{\eta_{1}}\right)\right)$ avec $\operatorname{Br} Y_{\eta_{1}}$ se réduit à $\operatorname{Br} \Delta_{\eta_{1}}=\operatorname{Br} K_{1}$. Toutes les fibres de $g_{1}$ sont scindées par la propriété (c) du lemme 5 , vu que le point générique $\eta_{1}$ de $\mathbf{P}_{k}^{n-1}$ est dans $U$. Pour chaque point fermé $\lambda$ de $\mathbf{P}_{K_{1}}^{1}$, la fibre de $g_{1}$ en $\lambda$ contient un ouvert non vide $W_{\lambda}$ qui est géométriquement intègre sur le corps résiduel $K_{\lambda}$ de $\lambda$. Soit $F_{\lambda}$ le corps des fonctions de $W_{\lambda}$, alors la restriction $H^{1}\left(K_{\lambda}, \mathbf{Q} / \mathbf{Z}\right) \rightarrow H^{1}\left(F_{\lambda}, \mathbf{Q} / \mathbf{Z}\right)$ est injective. Ceci implique qu'un élément $\beta$ de $\operatorname{Br} K$ dont l'image dans $\operatorname{Br} Y_{\eta}$ est dans $\operatorname{Br} Y_{\eta_{1}}$ était déjà non ramifié en tout point de $\Delta_{\eta_{1}}$ puisqu'il doit être non ramifié au point générique de $W_{\lambda}$. Ainsi un tel $\beta$ est dans $\operatorname{Br} K_{1}$, comme annoncé.

Maintenant comme le groupe $B$ est fini modulo $\mathrm{Br} K$, le lemme en découle.

Comme $C \subset \operatorname{Br} Y_{\eta_{1}}$ est fini modulo $\operatorname{Br} K_{1}$, on peut supposer (quitte à restreindre $U$ ) que la spécialisation $C_{m}$ de $C$ dans $\operatorname{Br} Y_{m} / \operatorname{Br}(k(m))$ est bien définie pour tout $m$ de $U$. D'autre part les 


\section{HARARI}

éléments de $B$ sont non ramifiés sur $Y_{\eta}$ et $B / \mathrm{Br} K$ est fini; on peut donc également (quitte à rétrécir $U)$ spécialiser les éléments de $B / \mathrm{Br} K$ au-dessus du point générique $\eta_{m}$ de la droite projective $\Delta_{m}$. On obtient un sous-groupe $B_{m}$ de $\operatorname{Br} Y_{\eta_{m}} / \operatorname{Br}\left(k(m)\left(\Delta_{m}\right)\right.$ ), où $Y_{\eta_{m}}$ est la fibre générique du morphisme $g_{m}: Y_{m} \rightarrow \Delta_{m}$. On a le diagramme suivant.

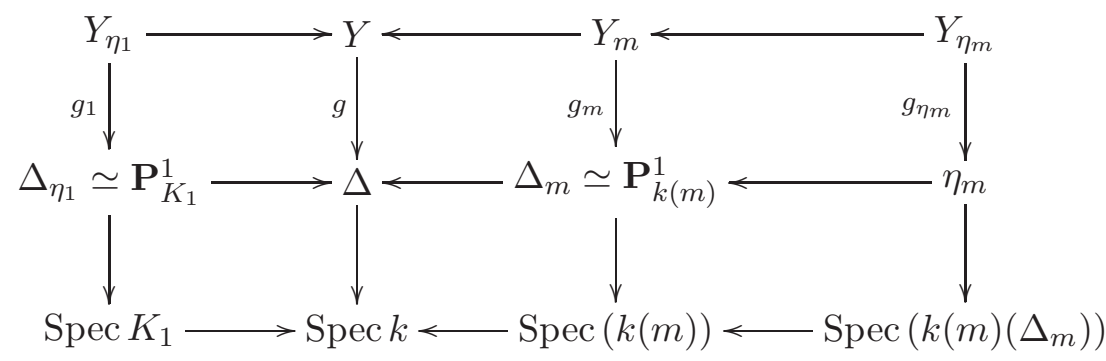

La proposition suivante joue un rôle-clé. L'idée est qu'elle permet de contrôler, à partir du seul groupe $C$, les spécialisations des éléments de $B$ qui, à un élément de $\operatorname{Br}\left(k\left(\Delta_{m}\right)\right)$ près, sont non ramifiées dans une $k$-fibre $Y_{m}$ de $Y \rightarrow \mathbf{P}_{k}^{n-1}$; ceci fait ensuite fonctionner la récurrence.

Proposition 2. Il existe un sous-ensemble hilbertien $H_{2}$ de $U(k) \subset \mathbf{P}_{k}^{n-1}(k)$ tel que pour tout $m \in H_{2}$, le groupe $\widetilde{B}_{m}:=\left[B_{m}+\operatorname{Br}\left(k\left(\Delta_{m}\right)\right)\right] \cap \operatorname{Br} Y_{m}$ soit inclus dans $C_{m}+\operatorname{Br} k$.

Remarque. Même si $B_{m}$ n'est défini que modulo $\operatorname{Br}\left(k\left(\Delta_{m}\right)\right)$ et $C_{m}$ modulo $\operatorname{Br} k, B_{m}+\operatorname{Br}\left(k\left(\Delta_{m}\right)\right)$ est bien défini comme sous-groupe de $\operatorname{Br} Y_{\eta_{m}}$ et $C_{m}+\operatorname{Br} k$ comme sous-groupe de $\operatorname{Br} Y_{m}$.

Preuve du théorème 1. Supposons pour l'instant que la proposition 2 soit démontrée. Comme le $k$-morphisme $\pi_{2}: \Delta \rightarrow \mathbf{P}_{k}^{n}$ est birationnel, le sous-ensemble $H^{\prime}:=\pi_{2}^{-1}(H)$ de $\Delta$ est hilbertien. Sa projection $\pi_{1}\left(H^{\prime}\right)$ est donc un sous-ensemble hilbertien de $\mathbf{P}_{k}^{n-1}(k)$. Quitte à remplacer $H_{2}$ par $H_{2} \cap \pi_{1}\left(H^{\prime}\right)$, nous pouvons supposer que $H_{2} \subset \pi_{1}\left(H^{\prime}\right)$, c'est-à-dire que pour tout point $m$ de $H_{2}$, la droite $\Delta_{m}$ rencontre $H^{\prime}$.

On applique alors l'hypothèse de récurrence au $k$-morphisme $f_{1}: Y \rightarrow \mathbf{P}_{k}^{n-1}$ et au sous-groupe $C$ de $\operatorname{Br} Y_{\eta_{1}}$ (remarquons que $Y_{\eta_{1}}$ est la fibre générique de $f_{1}$; elle est géométriquement intègre d'après le lemme 5(c), et lisse car $Y$ est lisse). Nous sommes bien dans les conditions d'application du théorème 1 car $f_{1}$ est projectif, surjectif, et les fibres de $f_{1}$ sont scindées d'après le lemme $5(\mathrm{~b})$; on vient également de voir que $C$ est fini modulo $\mathrm{Br} K_{1}$. On en déduit donc qu'il existe un point rationnel $m \in H_{2}$ et un point $\left(Q_{v}\right)$ de $Y_{m}\left(\mathbf{A}_{k}\right)^{C_{m}}$ (arbitrairement proche de $M_{v}$ pour $v$ dans $\left.S_{1}\right)$. On sait déjà par le lemme $5(\mathrm{c})$ que $Y_{m}$ est géométriquement intègre et on peut également supposer $Y_{m}$ lisse vu que $Y_{\eta_{1}}$ est lisse. Mais d'après la proposition 2 , on a aussi $\left(Q_{v}\right) \in Y_{m}\left(\mathbf{A}_{k}\right)^{\widetilde{B}_{m}}$, avec $\widetilde{B}_{m}=\left[B_{m}+\operatorname{Br}\left(k\left(\Delta_{m}\right)\right)\right] \cap \operatorname{Br} Y_{m}$. Le $k$-morphisme $g_{m}: Y_{m} \rightarrow \Delta_{m} \simeq \mathbf{P}_{k}^{1}$ est projectif, surjectif, à fibres scindées (car $m \in U$ ), et à fibre générique géométriquement intègre (lemme $5(\mathrm{c})$ ). Nous pouvons donc lui appliquer le cas $n=1$ du théorème 1 (i.e. le théorème 2) avec le groupe $B_{m}+\operatorname{Br}\left(k\left(\Delta_{m}\right)\right)$, qui est fini modulo $\operatorname{Br}\left(k\left(\Delta_{m}\right)\right)$ puisque $B$ était fini modulo $\operatorname{Br} K$.

Maintenant d'après le lemme 1 , on sait que $\Delta_{m}(k) \cap H^{\prime}$ (qui est non vide) est un sous-ensemble hilbertien de $\Delta_{m}(k)$. Posons alors $H_{1}=\Delta_{m}(k) \cap H^{\prime}$, on a donc $\pi_{2}\left(H_{1}\right) \subset H$. D'après le théorème 2 , on trouve un point $\theta_{1}$ dans $H_{1}$ tel que la fibre $\left(Y_{m}\right)_{\theta_{1}}$ de $Y_{m}$ en $\theta_{1}$ soit lisse et possède un point

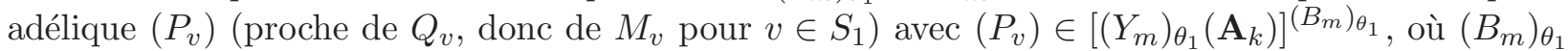
est la spécialisation de $B_{m}$ en $\theta_{1}$. Soit $\theta=\pi_{2}\left(\theta_{1}\right)$. Alors $\theta \in H$ et la fibre $Y_{\theta}$ de $p$ en $\theta$ n'est autre que $\left(Y_{m}\right)_{\theta_{1}}$ avec $\left(B_{m}\right)_{\theta_{1}}=B_{\theta}$ dans $\operatorname{Br} Y_{\theta} / \operatorname{Br} k$. Ainsi nous avons bien trouvé $\left(P_{v}\right)$ dans $Y_{\theta}\left(\mathbf{A}_{k}\right)^{B_{\theta}}$, proche de $M_{v}$ pour $v \in S_{1}$, avec $\theta$ dans l'ensemble hilbertien $H \subset \mathbf{P}_{k}^{n}(k)$ qu'on s'était donné.

Pour conclure la preuve du théorème 1 , il ne reste plus qu'à démontrer la proposition 2. 


\section{SpÉCiAlisation DES CONDitions DE MANin}

\subsection{Preuve de la proposition 2}

On considère encore le morphisme $g_{1}: Y_{\eta_{1}} \rightarrow \Delta_{\eta_{1}} \simeq \mathbf{P}_{K_{1}}^{1}$, dont la fibre générique est $Y_{\eta}$. Soient $\alpha \in \operatorname{Br} Y_{\eta}$ et $\lambda$ un point fermé de $\Delta_{\eta_{1}}$, puis $\eta_{\lambda}$ un point de codimension 1 (fixé) de $Y_{\eta_{1}}$ contenu dans la fibre de $g_{1}$ en $\lambda$. Soient $F_{\lambda}$ le corps résiduel de $\eta_{\lambda}$ et $\partial(\alpha) \in H^{1}\left(F_{\lambda}, \mathbf{Q} / \mathbf{Z}\right)$ le résidu de $\alpha$ en $\eta_{\lambda}$. On fixe un ouvert $V_{\lambda}$ de la fibre en $\lambda$ de point générique $\eta_{\lambda}$ tel que $\partial(\alpha) \in H^{1}\left(V_{\lambda}, \mathbf{Q} / \mathbf{Z}\right)$. Quand le corps résiduel $K_{\lambda}$ de $\lambda$ est algébriquement clos dans $F_{\lambda}$, on dira que $\partial(\alpha)$ est géométrique s'il ne provient pas de $H^{1}\left(K_{\lambda}, \mathbf{Q} / \mathbf{Z}\right)$. Cette notion correspond grosso modo au fait qu'on ne peut pas rendre $\alpha$ non ramifié en le modifiant par un élément de $\operatorname{Br} K=\operatorname{Br}\left(K_{1}\left(\mathbf{P}_{K_{1}}^{1}\right)\right)$. Le lemme 7 ci-dessous montre que, grâce au théorème d'irréductibilité de Hilbert, cette propriété se spécialise bien. Ceci va permettre de montrer la proposition 2 en excluant de $\widetilde{B}_{m}$ les éléments obtenus (modulo $\operatorname{Br}\left(k\left(\Delta_{m}\right)\right)$ par spécialisation d'un tel $\alpha$.

Lemme 7. Avec les notations ci-dessus, il existe un sous-ensemble hilbertien $H_{\alpha}$ de $U(k) \subset \mathbf{P}_{k}^{n-1}(k)$ tel que pour tout $m$ de $H_{\alpha}$ on ait les propriétés suivantes.

(a) Le point $\lambda$ se spécialise en $m$ en un point fermé $\lambda_{m}$ de $\Delta_{m} \simeq \mathbf{P}_{k}^{1}$, et $V_{\lambda}$ en un ouvert intègre $V_{\lambda_{m}}$ (respectivement géométriquement intègre sur le corps résiduel $k\left(\lambda_{m}\right)$ de $\lambda_{m}$ si $K_{\lambda}$ était algébriquement clos dans $F_{\lambda}$ ) de la fibre de $g_{m}$ en $\lambda_{m}$.

(b) Si $\partial(\alpha)$ est non trivial, alors il se spécialise en un élément non trivial de $H^{1}\left(V_{\lambda_{m}}, \mathbf{Q} / \mathbf{Z}\right)$, qui est le résidu $\partial_{m}\left(\alpha_{m}\right)$ de $\alpha_{m}$ au point générique de $V_{\lambda_{m}}$. En particulier $\alpha_{m} \notin \operatorname{Br} Y_{m}$.

(c) Si de plus $\partial(\alpha)$ était géométrique, alors $\partial_{m}\left(\alpha_{m}\right)$ est géométrique, i.e. ne provient pas de $H^{1}\left(k\left(\lambda_{m}\right), \mathbf{Q} / \mathbf{Z}\right)$. On a alors $\alpha_{m} \notin\left[\operatorname{Br} Y_{m}+\operatorname{Br}\left(k\left(\Delta_{m}\right)\right)\right]$.

Remarques. Nous faisons les remarques suivantes.

(i) Un résidu non trivial existe pour tout élément de $\operatorname{Br} Y_{\eta}$ qui n'est pas dans $\operatorname{Br} Y_{\eta_{1}}$.

(ii) La condition (a) est indépendante de $\alpha$ et $\partial(\alpha)$.

(iii) Rappelons qu'il existe un ouvert non vide $U_{\alpha}$ de $\mathbf{P}_{k}^{n-1}$ tel que pour tout $m \in U_{\alpha}$, la spécialisation $\alpha_{m} \in \operatorname{Br} Y_{\eta_{m}}$ ait un sens, où $Y_{\eta_{m}}$ est la fibre générique de $g_{m}: Y_{m} \rightarrow \Delta_{m} \simeq \mathbf{P}_{k(m)}^{1}$.

Démonstration. Soient $F_{\lambda}^{\prime} / F_{\lambda}$ l'extension cyclique (non triviale si $\partial(\alpha)$ n'est pas nul) correspondant au résidu $\partial(\alpha)$, et $K_{\lambda}^{\prime}$ la fermeture algébrique du corps résiduel $K_{\lambda}$ de $\lambda$ dans $F_{\lambda}^{\prime}$. On fixe une extension transcendante pure $F_{1}$ de $K_{1}$ tel que $F_{\lambda}$ soit fini sur $F_{1}$.

On étend alors la situation au-dessus d'un ouvert non vide $U$ (on peut supposer que c'est le même que celui de l'énoncé du lemme 7 , quitte à restreindre) de $\mathbf{P}_{k}^{n-1}$; c'est-à-dire qu'on fixe des variétés $\mathcal{U}_{\lambda}, \mathcal{U}_{\lambda}^{\prime}, \mathcal{V}_{1}, \mathcal{V}_{\lambda}, \mathcal{V}_{\lambda}^{\prime}$, équipées de $k$-morphismes vers $U$, de corps des fonctions respectifs $K_{\lambda}, K_{\lambda}^{\prime}, F_{1}, F_{\lambda}, F_{\lambda}^{\prime}$. On peut prendre $\mathcal{V}_{1}$ isomorphe à un ouvert de $\mathbf{A}_{k}^{r}$ avec $r \geqslant n-1$, d'où une projection $\mathcal{V}_{1} \rightarrow U$. On suppose également que $\mathcal{V}_{\lambda}^{\prime} / \mathcal{V}_{1}$ est un revêtement étale, que l'on a $\partial(\alpha) \in H^{1}\left(\mathcal{V}_{\lambda}^{\prime} / \mathcal{V}_{\lambda}, \mathbf{Q} / \mathbf{Z}\right)$, et que pour $m \in U$, l'élément $\alpha$ se spécialise en $\alpha_{m} \in \operatorname{Br} Y_{\eta_{m}}$. Enfin, si on fait en plus l'hypothèse que $K_{\lambda}$ est algébriquement clos dans $F_{\lambda}$, on peut supposer que pour $m \in U$, la spécialisation $V_{\lambda_{m}}$ de la $K_{\lambda}$-variété géométriquement intègre $\mathcal{V}_{\lambda}$ reste géométriquement intègre sur $k\left(\lambda_{m}\right)$ dès que $\lambda_{m}$ reste un point fermé de $\Delta_{m}$.

D'après le théorème d'irréductibilité de Hilbert, il existe un ensemble hilbertien $H_{3} \subset \mathcal{V}_{1}(k)$ tel que pour tout point $m^{\prime}$ dans $H_{3}$, la fibre de $\mathcal{V}_{\lambda}^{\prime} / \mathcal{V}_{1}$ en $m^{\prime}$ soit intègre. On dispose également d'un sous-ensemble hilbertien $H_{3}^{\prime}$ de $U(k)$ tel que pour tout $m$ dans $H_{3}^{\prime}$, la fibre de $\mathcal{U}_{\lambda}^{\prime} / U$ en $m$ soit intègre. Soit alors $H_{\alpha}$ l'intersection de $H_{3}^{\prime}$ avec la projection de $H_{3}$ sur $U$. Pour $m \in H_{\alpha}$, la spécialisation $\lambda_{m}$ reste un point (fermé) de $\Delta_{m}$ (parce que $m \in H_{3}^{\prime}$ ) et $V_{\lambda_{m}}$ reste intègre (parce que $m$ est la projection d'un point de $H_{3}$ ). Si en outre $K_{\lambda}$ était algébriquement clos dans $F_{\lambda}$ (i.e. $V_{\lambda}$ géométriquement intègre sur $K_{\lambda}$ ), on obtient en plus que $V_{\lambda_{m}}$ est géométriquement intègre sur $k\left(\lambda_{m}\right)$ d'après les propriétés de $U$. Ainsi $\alpha_{m}$ possède au point générique de $V_{\lambda_{m}}$ un résidu $\partial_{m}\left(\alpha_{m}\right)$ 


\section{HARARI}

qui correspond à la spécialisation de $\mathcal{V}_{\lambda}^{\prime} / \mathcal{V}_{\lambda}$ en $m$. D'après le choix de $H_{3}$, le résidu $\partial_{m}\left(\alpha_{m}\right)$ reste non trivial si $\partial(\alpha) \neq 0$, donc $\alpha_{m} \notin \operatorname{Br} Y_{m}$. D'où (a) et (b).

Si en outre le résidu $\partial(\alpha)$ était géométrique, on voit que le résidu $\partial_{m}\left(\alpha_{m}\right)$ l'est encore puisque l'extension $F_{\lambda}^{\prime} / K_{\lambda}^{\prime} F_{\lambda}$ était alors non triviale, donc aussi sa spécialisation vu le choix de $H_{3}$. De ce fait, le résidu correspondant de $\alpha_{m}+\beta$ ne peut pas être nul si $\beta \in \operatorname{Br}\left(k\left(\Delta_{m}\right)\right)$ puisque le résidu de $\beta$ provient alors de $\left.H^{1}\left(k\left(\lambda_{m}\right), \mathbf{Q} / \mathbf{Z}\right)\right)$. Ceci prouve (c).

On peut maintenant terminer la preuve de la proposition 2. Comme les fibres de $g_{1}: Y_{\eta_{1}} \rightarrow$ $\Delta_{\eta_{1}} \simeq \mathbf{P}_{K_{1}}^{1}$ sont scindées, on peut choisir pour tout point fermé $\lambda$ de $\mathbf{A}_{K_{1}}^{1} \subset \Delta_{\eta_{1}}$ un ouvert non vide $W_{\lambda}$ (fixé dans toute la suite) de la fibre en $\lambda$ qui est géométriquement intègre sur le corps résiduel $K_{\lambda}$ de $\lambda$. Soit $\Gamma=\left\{\alpha_{1}, \ldots, \alpha_{r}\right\}$ un sous-groupe fini de $B$ qui se surjecte sur $B / \operatorname{Br} K$. Quitte à modifier certains éléments de $\Gamma$ par des éléments de $\operatorname{Br} K$, on peut supposer (via la suite exacte de Faddeev pour $\left.\operatorname{Br}\left(K_{1}\left(\mathbf{A}_{K_{1}}^{1}\right)\right)=\operatorname{Br} K\right)$ que tout élément $\alpha$ de $\Gamma$ vérifie l'alternative :

(i) $\alpha$ a un résidu géométrique au point générique de $W_{\lambda}$ pour un certain point fermé $\lambda$ de $\mathbf{A}_{K_{1}}^{1}$ (on appellera $\Gamma_{1}$ l'ensemble de $\operatorname{ces} \alpha$ ); ou

(ii) pour tout point fermé $\lambda$ de $\mathbf{A}_{K_{1}}^{1}$, le résidu $\partial_{\lambda}(\alpha)$ de $\alpha$ au point générique de $W_{\lambda}$ est nul (on appellera $\Gamma_{2}$ l'ensemble de ces $\alpha$ ).

Soit $\Lambda$ un ensemble fini de points fermés de $\mathbf{A}_{K_{1}}^{1}$ tel que tous les éléments de $\Gamma$ soient non ramifiés sur l'ouvert complémentaire $V_{0}$ des fibres de $g_{1}$ au-dessus de $\Lambda$ et de $\infty$. On choisit un ouvert non vide $U_{0} \subset U$ de $\mathbf{P}_{k}^{n-1}$ tel que $V_{0}$ s'étende en un $\mathcal{V}_{0}$ lisse au-dessus de $U_{0}$, et on demande en plus qu'on ait $\alpha \in \operatorname{Br} \mathcal{V}_{0}$ pour tout $\alpha$ de $\Gamma$. Ainsi si $m \in U_{0}$, les éléments de $\Gamma$ se spécialisent en $m$ en des éléments non ramifiés sur la fibre de $\mathcal{V}_{0}$ en $m$.

Appliquons le lemme 7. Le (a) donne déjà un ensemble hilbertien $H_{0} \subset U(k)$ tel que pour $m \in H_{0}$, la spécialisation de chaque $\lambda$ de $\Lambda$ reste un point fermé $\lambda_{m}$ de $\Delta_{m} \simeq \mathbf{P}_{k}^{1}$, et la spécialisation $W_{\lambda_{m}}$ de $W_{\lambda}$ reste géométriquement intègre sur $\lambda_{m}$ (on intersecte les différents ensembles hilbertiens obtenus pour chaque $W_{\lambda}$ ). Le (b) nous donne, pour chaque élément $\alpha$ de $\Gamma$ qui n'est pas dans $\operatorname{Br} Y_{\eta_{1}}$, un ensemble hilbertien $H_{\alpha} \subset U(k)$ tel que pour $m \in H_{\alpha}$, la spécialisation $\alpha_{m}$ ne soit pas dans $\operatorname{Br} Y_{m}$. D'après le (c) on a même $\alpha_{m} \notin \operatorname{Br} Y_{m}+\operatorname{Br}\left(k\left(\Delta_{m}\right)\right)$ si $\alpha \in \Gamma_{1}$.

On remarque que les $\alpha$ de $\Gamma$ qui sont dans $\operatorname{Br} Y_{\eta_{1}}$ sont dans $C$ par définition de $C$. Soit $H_{2}$ le sous-ensemble hilbertien de $\mathbf{P}_{k}^{n-1}$ obtenu en prenant l'intersection de $U_{0} \cap H_{0}$ et de tous les $H_{\alpha}$ définis plus haut. Soient $m \in H_{2}$ et $\gamma$ un élément de $\widetilde{B}_{m}$. Par définition de $\widetilde{B}_{m}$, il existe $\alpha \in \Gamma$ et $\beta \in \operatorname{Br}\left(k\left(\Delta_{m}\right)\right)$ tels que $\gamma=\alpha_{m}+\beta$ dans $\operatorname{Br} Y_{\eta_{m}}$. En particulier $\alpha_{m} \in \operatorname{Br} Y_{m}+\operatorname{Br}\left(k\left(\Delta_{m}\right)\right)$. Supposons qu'un tel $\alpha$ de $\Gamma$ ne soit pas dans $\operatorname{Br} Y_{\eta_{1}}$, et montrons qu'on aboutit à une contradiction. Comme $m \in H_{\alpha}$, le lemme 7 (c) nous dit déjà que $\alpha \in \Gamma_{2}$. Ainsi pour tout $\lambda \in \Lambda, \alpha_{m}$ est non ramifié au point générique de $W_{\lambda_{m}}$ (qui est géométriquement intègre). Comme $\alpha_{m}+\beta$ est non ramifié sur $Y_{m}$, ceci impose que $\beta$ soit également non ramifié en $\lambda_{m}$. D'autre part $\alpha_{m}$ était non ramifié sur la spécialisation de $\mathcal{V}_{0}$, donc en dehors des fibres de $g_{m}: Y_{m} \rightarrow \Delta_{m}$ au-dessus des $\lambda_{m}$ et de $\infty$; ainsi $\beta$ également car toutes les fibres de $g_{m}$ sont scindées. Finalement $\beta$ est non ramifié en tout point fermé de $\mathbf{A}_{k}^{1}$, donc $\beta \in \operatorname{Br} k$. Mais alors $\alpha_{m} \in \operatorname{Br} Y_{m}$ ce qui contredit le lemme $7(\mathrm{~b})$.

Ainsi $\alpha \in \operatorname{Br} Y_{\eta_{1}}$, d'où $\alpha \in C$ et $\alpha_{m} \in \operatorname{Br} Y_{m}$. Comme $\gamma=\alpha_{m}+\beta$ est également dans $\operatorname{Br} Y_{m}$, on obtient que $\beta$ est dans $\operatorname{Br} Y_{m} \cap \operatorname{Br}\left(k\left(\Delta_{m}\right)\right.$ ), donc dans $\operatorname{Br} k$ (toujours parce que toutes les fibres de $g_{m}$ sont scindées). On a donc bien $\gamma \in C_{m}+\mathrm{Br} k$, ce qui achève la preuve de la proposition 2 , et par voie de conséquence du théorème 1.

\section{REMERCIEMENTS}

Ce travail est né à la suite de longues et fructueuses discussions avec J.-L. Colliot-Thélène, et a également bénéficié de ses commentaires; je l'en remercie chaleureusement. Je remercie également 


\section{SpÉCiAlisation DES CONDitions DE MANin}

O. Wittenberg pour m'avoir tenu au courant de ses recherches sur les intersections de deux quadriques [Wit07] qui ont motivé le présent article, et le rapporteur anonyme pour ses nombreuses suggestions pertinentes. Enfin je tiens à remercier l'Institut Alfréd Rényi, et plus particulièrement T. Szamuely, pour m'avoir permis de passer un mois à Budapest, pendant lequel cet article a été conçu.

\section{BIBLIOGRAPHIE}

BS01 A. Bender and P. Swinnerton-Dyer, Solubility of certain pencils of curves of genus 1, and of the intersection of two quadrics in $\mathbf{P}^{4}$, Proc. London Math. Soc. (3) 83 (2001), 299-329.

Col01 J.-L. Colliot-Thélène, Hasse principle for pencils of curves of genus one whose Jacobians have a rational 2-division point, close variation on a paper of Bender and Swinnerton-Dyer, in Rational points on algebraic varieties, Progress in Mathematics, vol. 199 (Birkhäuser, Basel, 2001), 117-161.

Col03 J.-L. Colliot-Thélène, Points rationnels sur les fibrations, in Higher dimensional varieties and rational points, Bolyai Society Mathematical Studies, vol. 12 (Springer, Berlin, 2003), 171-221.

CSS98 J.-L. Colliot-Thélène, A. N. Skorobogatov and P. Swinnerton-Dyer, Hasse principle for pencils of curves of genus one whose Jacobians have rational 2-division points, Invent. Math. 134 (1998), $579-650$.

Eke90 T. Ekedahl, An effective version of Hilbert's irreducibility theorem, in Séminaire de théorie des nombres de Paris 1988-1989, Progress in Mathematics, vol. 91, ed. C. Goldstein (Birkhäuser, Basel, 1990), 241-248.

Gro68 A. Grothendieck, Le groupe de Brauer III, exemples et compléments, in Dix exposés sur la cohomologie des schémas (Masson-North Holland, Amsterdam, 1968).

Har94 D. Harari, Méthode des fibrations et obstruction de Manin, Duke Math. J. 75 (1994), 221-260.

Har97 D. Harari, Flèches de spécialisation en cohomologie étale et applications arithmétiques, Bull. Soc. math. France 125 (1997), 143-166.

Jou83 J.-P. Jouanolou, Théorèmes de Bertini et applications, Progress in Mathematics, vol. 42 (Birkhäuser, Boston, MA, 1983).

Ser94 J.-P. Serre, Cohomologie Galoisienne, fifth edition (Springer, Berlin, 1994).

Sko01 A. N. Skorobogatov, Torsors and rational points (Cambridge University Press, Cambridge, 2001).

Wit07 O. Wittenberg, Intersection de deux quadriques et pinceaux de courbes de genre 1, Lecture Notes in Mathematics, vol. 1901 (Springer, 2007).

Wit06 O. Wittenberg, Principe de Hasse pour les intersections de deux quadriques, C. R. Math. Acad. Sci. Paris 342 (2006), 223-227.

David Harari David.Harari@math.u-psud.fr

Laboratoire de Mathématiques, Université Paris-Sud, Bâtiment 425, 91405 Orsay cedex, France 